\title{
How to interpret recent restrictive transfusion trials in cardiac surgery: More new data or new more data?
}

\author{
Jerrold H. Levy, MD, FAHA, FCCM, ${ }^{a}$ and Marie E. Steiner, MD, MS ${ }^{b}$
}

\footnotetext{
From the a Departments of Anesthesiology, Critical Care, and Surgery, Duke University School of Medicine, Durham, NC; and ${ }^{\mathrm{b}}$ Department of Hematology and Pediatrics, University of Minnesota School of Medicine, Minneapolis, Minn.

Funded by the Department of Anesthesiology, Duke University School of Medicine, Durham, NC.

Received for publication Oct 22, 2018; accepted for publication Oct 23, 2018; available ahead of print Dec 6, 2018 .

Address for reprints: Jerrold H. Levy, MD, FAHA, FCCM, Duke University Medical Center, 2301 Erwin Rd, 5691H HAFS, Box 3094, Durham, NC 27710 (E-mail: jerrold.levy@ duke.edu).

J Thorac Cardiovasc Surg 2019;157:1038-40

$0022-5223 / \$ 36.00$

Copyright $(2) 2018$ by The American Association for Thoracic Surgery

https://doi.org/10.1016/j.jtcvs.2018.10.108
}

Patient blood management strategies have increasingly become an area of focus for clinicians to reduce allogeneic blood exposure. ${ }^{1}$ There are multiple reasons to reduce blood exposures to decrease costs, risks, and antigenic exposure. Algorithms for blood product administration are the basis for most strategies and routinely specifically state indications and thresholds for blood product admnistration. ${ }^{2}$ Of all the hospitalized patients, cardiac surgical patients are at significant risk for bleeding and transfusion because of the complexities of the surgery, activation of the multiple coagulation and inflammatory pathways, dilutional changes, hypothermia, and extracorporeal activation. ${ }^{3}$ Of note, cardiac surgical patients represent a major group of patients who are recipients of blood used in hospitals.

The Transfusion Requirement in Critical Care (TRICC) trial evaluated a liberal versus restrictive red cell transfusion strategy in 838 patients in the intensive care unit (ICU) and showed no mortality difference, but it excluded cardiac surgical patients. ${ }^{5}$ A subgroup analysis of TRICC including patients with cardiovascular diagnoses but not surgical interventions showed no overall mortality differences between liberal and restrictive strategies, with less deterioration in multiple organ dysfunction in the restrictive group. ${ }^{6}$ The evidence in patients after cardiac surgery was limited by small, poor-quality trials. The concern has been that a restrictive strategy may increase the risk of anemia-induced tissue hypoxia. Mazer and colleagues ${ }^{7,8}$ have now evaluated this concern in 2 reports: the Transfusion Requirements in Cardiac Surgery (TRICS) II and TRICS III. The question of whether a restrictive or liberal approach to transfusion in cardiac surgical patients who have a moderate-to-high risk of mortality affects a composite outcome of cardiovascular events, renal failure requiring dialysis, or death as follows.

In TRICS II, Mazer and colleagues ${ }^{7}$ performed a randomized noninferiority trial of 5243 adult patients

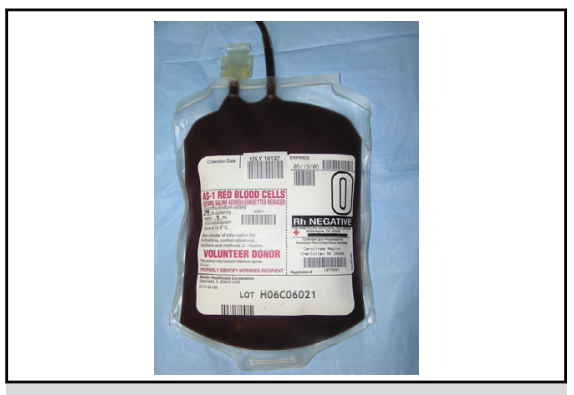

The TRICS trials

Central Message

The TRICS trials provide more data for hemoglobin "triggers" to guide transfusions; however, future efforts should be directed at generating new data using physiologic and other end points

See Commentaries on pages 1041 and 1043.

undergoing cardiac surgery with a European System for Cardiac Operative Risk Evaluation I of 6 or more. With this scoring system, higher scores are consistent with a higher mortality risk. Patients were randomized to a restrictive red blood cell (RBC) threshold for transfusion for a hemoglobin of less than $7.5 \mathrm{~g} / \mathrm{dL}$ or an RBC transfusion threshold of less than $9.5 \mathrm{~g} / \mathrm{dL}$ if in the ICU or during surgery and less than $8.5 \mathrm{~g} / \mathrm{dL}$ when out of the ICU. The primary outcome was a composite of all-cause mortality, myocardial infarction, stroke, or dialysis-dependent acute renal failure up to the time of discharge from the hospital or 28 days postoperatively. The secondary outcomes were clinical outcomes that included RBC transfusions.

In the study, there were coronary artery bypass grafts only in $26 \%$ of patients, coronary artery bypass graft + valve in $19 \%$ of patients, and valve surgery in $29 \%$ of the patients, and approximately $12 \%$ reoperations in both groups with mean cardiopulmonary bypass times of approximately 120 minutes. Tranexamic acid was administered in approximately $91 \%$ of the patients. The composite primary outcome was $11.4 \%$ of the restrictive patients compared with $12.5 \%$ of patients in the liberal group with an odds ratio (OR) of 0.90 and a reduction in mortality of $3.0 \%$ compared with $3.6 \%$ (OR, 0.85). Specific outcomes included myocardial infarction in $5.9 \%$ versus $5.9 \%$, stroke in $1.9 \%$ versus $2.0 \%$, and new renal failure 
in $2.5 \%$ versus $3.0 \%$ (restrictive vs liberal). As expected, the restrictive transfusion group had RBCs transfused in $52.3 \%$ compared with $72.6 \%$ in the liberal group, but without any additional differences in the secondary outcomes. On subgroup analysis, the primary end point was significantly reduced in patients aged 75 years or more $(P$ for interaction $=.004$ ).

Secondary outcomes for restrictive versus liberal transfusion strategies included plasma transfusion $(23.5 \%$ vs $27.1 \%, P<.05$ ), median length of stay ( 8 days vs 8 days, $P<.05)$, ICU median length of stay ( 2.1 vs $1.9, P<.05)$, and infection $(5.0 \%$ vs $4.2 \%, P>.05)$.

In Mazer and colleagues' follow-up study TRICS III, ${ }^{8}$ they report additional clinical outcomes 6 months after surgery in their 5243 adult patients. The strictly defined primary composite outcome occurred in $17.4 \%$ (402 of 2317 ) in the restrictive group compared with $17.1 \%$ (402 of 2347) in the liberal group (OR, 1.02) with a $P$ value of .006 for noninferiority, and mortality was $6.2 \%$ compared with $6.4 \%$, respectively (OR, 0.95), again without differences in secondary outcomes. ${ }^{9}$ The authors concluded that in moderate- to high-risk cardiac surgical patients, an RBC transfusion restrictive strategy was noninferior to a liberal strategy for their primary and secondary outcomes at 6 months after surgery. The primary outcome, including all-cause mortality, nonfatal myocardial infarction (MI), stroke, new-onset renal failure with dialysis, and betweenhospital admission and discharge/28 days, for restrictive versus liberal transfusion strategies was $11.4 \%$ versus $12.5 \%$ (OR, 0.90; 95\% confidence interval, 0.76-1.07; $P$ for noninferiority $<.001){ }^{8}$

This follow-up trial confirms earlier observations that blood transfusions to an arbitrary higher threshold may not always be beneficial and could be associated with potential harm. A more restrictive strategy appeared to be particularly beneficial among elderly patients. These findings will likely influence future perioperative guidelines. However, it is important to note that patients undergoing heart transplantation or ventricular assist device insertion, and pregnant women were excluded.

Overall, the strengths of this large, multicenter study with appropriate follow-ups and analyses included blinding of investigators who are assessing primary outcomes. However, important limitations include the nonblinded clinical staff administering the blood, and the per-protocol analysis excluded patients when adherence to the protocol was less than $90 \%$, a critical finding that has important potential to remove sicker patients out of the analysis of the primary outcome that could potentially influence the power of the study. However, the study included a modified intentionto-treat analysis that did include these patients and still demonstrated noninferiority in the restrictive strategy. The results also may be limited by the transfusion thresholds themselves that were established through pilot and consensus data if the 2 thresholds are too close to identify a true difference in outcomes. ${ }^{9}$ The New England Journal of Medicine embraces large, well-conducted clinical studies as thoughtfully performed by the investigators, and as an example previously reported significant results with tranexamic acid despite multiple prior smaller studies that may have shown equipoise. ${ }^{10}$ However, TRICS III, as all similar studies, focuses on the hemoglobin value without potentially better defining a clinically meaningful parameter to examine what should be the appropriate clinically indicated or other meaningful threshold for RBC transfusion. Unfortunately, one of the issues that studies addressing specific hemoglobin thresholds do not examine is to base their interventions on precise measures of oxygen delivery or oxygen debt. This is because we do not have good measures to examine these important physiologic indices, and as a result, we therefore treat numeric values of hemoglobin rather than define an appropriate physiologic threshold for each particular patient. Again, we, unfortunately, do not have the ability to readily measure specific oxygen delivery or oxygen debt, and as a result treat specific hemoglobin thresholds instead.

In the modern era of highly protocolized perioperative management, we often routinely chase numeric values such as hemoglobin levels without always closely evaluating patients. Are there physiologic differences between a hemoglobin of $7.5 \mathrm{~g} / \mathrm{dL}$ versus $9 \mathrm{~g} / \mathrm{dL}$ in our cardiac surgical patients? Patients are often lying in bed, may or may not be mobilized, are often not physically active, and may be receiving mechanical respiratory support and ride in wheelchairs upon hospital discharge. When they go home, they may remain relatively immobile without encouragement. Is the restrictive threshold in cardiac surgery patients mitigated by inactivity and reduced oxygen demand? As previously stated, in the TRICC trial, the clinical staff was not blinded and the protocol analysis excluded patients in whom adherence to the protocol was less than $90 \%$, a critical consideration that may have removed more complex critically ill patients from the analysis of the primary outcome that could potentially influence the power of the study.

Clinical trials that focus on RBC transfusions increase hemoglobin levels, but oxygen delivery to tissues may not be increased because of multiple factors. ${ }^{11}$ The TRICC trial is one more study that continues to demonstrate that using restrictive thresholds for transfusions of RBCs are noninferior to liberal thresholds in most clinical scenarios. ${ }^{1}$ As noted from the 2015 Proceedings of the National Heart, Lung, and Blood Institute's State of the Science in Transfusion Medicine Symposium, decisions to transfuse RBCs ideally should be based on the extent to which anemia contributes to tissue oxygen delivery. ${ }^{11}$ In addition, the recent recommendations from a pediatric critical care consensus initiative calls for further study of physiologic thresholds 
and alternatives to optimize oxygen delivery/consumption balance. ${ }^{12}$ We believe that further studies should identify specific physiologic end points for outcomes to decide on the thresholds for transfusion. This concept necessitates developing ways to determine the relationship of transfusion to tissue oxygen delivery, especially in critically ill patients.

There are also other important unanswered questions regarding transfusions of other components in cardiac surgical patients that need to be addressed in future studies. Despite our extensive routine use of platelet transfusions in our patients, there is no high-quality evidence to determine the appropriateness of platelet transfusions with bleeding. ${ }^{13,14}$ Most transfusion algorithms in cardiac surgical patients use platelet counts as the laboratory determinant for platelet transfusions. Rotational thromboelastometry and thromboelastography, both viscoelastic tests, are often part of transfusion algorithms, but most algorithms, in general, reduce allogenic blood product administration because they prevent the empiric use of blood products without specific coagulation abnormalities. ${ }^{15}$ Despite the increasing availability of platelet function testing, in bleeding and coagulopathy, many of the platelet function tests currently available require specific platelet numbers, do not work in anemic patients, or have other limiting factors. As noted in a recent National Heart, Lung, and Blood Institute review, there are no current standards for clinical or laboratory assessment of the hemostatic efficacy of platelet administration in bleeding patients. ${ }^{11}$ Clinical trials are needed that validate clinical bleeding measurements, evaluate the usefulness of in vitro testing, and assess the adverse effects of platelet transfusion. ${ }^{11}$

Have we reached clinical equipoise regarding additional studies for transfusion triggers of hemoglobin in cardiac surgery? We believe so, and as a result, if future studies are to be conducted, then physiologic thresholds and parameters need to be thoughtfully considered in the design of future studies. Based on the title of this commentary on how to interpret recent restrictive transfusion trials in cardiac surgery, the TRICS trials provide more data using hemoglobin "triggers" to guide transfusion in cardiac surgical patients. However, future efforts should be directed at generating new data using physiologic and other end points, and include all patients in their studies as currently being performed in the pragmatic trial design of the FIBrinogen REplenishment in Surgery study in Canada in cardiac surgical patients evaluating fibrinogen versus cryoprecipitate for bleeding. ${ }^{16}$

\section{Conflict of Interest Statement}

J.H.L.: Steering Committees for CSL Behring, Instrumentation Labs, Merck, and Octapharma. M.E.S. has nothing to disclose with regard to commercial support.

\section{References}

1. Goodnough LT, Levy JH, Murphy MF. Concepts of blood transfusion in adults. Lancet. 2013;381:1845-54.

2. Steiner ME, Despotis GJ. Transfusion algorithms and how they apply to blood conservation: the high-risk cardiac surgical patient. Hematol Oncol Clin North Am. 2007;21:177-84.

3. Levy JH, Dutton RP, Hemphill JC III, Shander A, Cooper D, Paidas MJ, et al. Multidisciplinary approach to the challenge of hemostasis. Anesth Analg. 2010;110:354-64.

4. Bennett-Guerrero E, Zhao Y, O'Brien SM, Ferguson TB Jr, Peterson ED, Gammie JS, et al. Variation in use of blood transfusion in coronary artery bypass graft surgery. JAMA. 2010;304:1568-75.

5. Hebert PC, Wells G, Blajchman MA, Marshall J, Martin C, Pagliarello G, et al. A multicenter, randomized, controlled clinical trial of transfusion requirements in critical care. Transfusion Requirements in Critical Care Investigators, Canadian Critical Care Trials Group. N Engl J Med. 1999;340:409-17.

6. Hebert PC, Yetisir E, Martin C, Blajchman MA, Wells G, Marshall J, et al. Is a low transfusion threshold safe in critically ill patients with cardiovascular diseases? Crit Care Med. 2001;29:227-34.

7. Mazer CD, Whitlock RP, Fergusson DA, Hall J, Belley-Cote E, Connolly K, et al. Restrictive or liberal red-cell transfusion for cardiac surgery. N Engl J Med. 2017; 377:2133-44.

8. Mazer CD, Whitlock RP, Fergusson DA, Belley-Cote E, Connolly K, Khanykin B, et al. Six-Month outcomes after restrictive or liberal transfusion for cardiac surgery. N Engl J Med. 2018;379:1224-33.

9. Shehata N, Whitlock R, Fergusson DA, Thorpe KE, MacAdams C, Grocott HP, et al. Transfusion Requirements in Cardiac Surgery III (TRICS III): study design of a randomized controlled trial. J Cardiothorac Vasc Anesth. 2018;32:121-9.

10. Myles PS, Smith JA, Forbes A, Silbert B, Jayarajah M, Painter T, et al. Tranexamic acid in patients undergoing coronary-artery surgery. $N$ Engl J Med. 2017; 376:136-48.

11. Spitalnik SL, Triulzi D, Devine DV, Dzik WH, Eder AF, Gernsheimer T, et al. 2015 proceedings of the National Heart, Lung, and Blood Institute's State of the Science in Transfusion Medicine symposium. Transfusion. 2015;55:2282-90.

12. Doctor A, Cholette JM, Remy KE, Argent A, Carson JL, Valentine SL, et al. Recommendations on RBC transfusion in general critically ill children based on hemoglobin and/or physiologic thresholds from the pediatric critical care transfusion and anemia expertise initiative. Pediatr Crit Care Med. 2018;19(9S Suppl 1):S98-113.

13. Levy JH, Rossaint R, Zacharowski K, Spahn DR. What is the evidence for platelet transfusion in perioperative settings? Vox Sang. 2017;112:704-12.

14. Kumar A, Mhaskar R, Grossman BJ, Kaufman RM, Tobian AA, Kleinman S, et al. Platelet transfusion: a systematic review of the clinical evidence. Transfusion. 2015;55:1116-27. quiz 5.

15. Weber CF, Gorlinger K, Meininger D, Herrmann E, Bingold T, Moritz A, et al. Point-of-care testing: a prospective, randomized clinical trial of efficacy in coagulopathic cardiac surgery patients. Anesthesiology. 2012;117:531-47.

16. Karkouti K, Callum J, Rao V, Heddle N, Farkouh ME, Crowther MA, et al. Protocol for a phase III, non-inferiority, randomised comparison of a new fibrinogen concentrate versus cryoprecipitate for treating acquired hypofibrinogenaemia in bleeding cardiac surgical patients: the FIBRES trial. BMJ Open. 2018;8: e020741. 